\title{
Palliative chemotherapy followed by methylation inhibitor in high-risk acute myeloid leukemia: An in vitro and clinical study
}

\author{
BINGJIE DING ${ }^{1 *}$, ZHIXIANG WANG $^{1 *}$, XUEJIE JIANG ${ }^{1}$, XIAODONG LI ${ }^{1}$, CHUNLI WANG $^{1}$, \\ QINGXIU ZHONG ${ }^{1,2}$, LING JIANG ${ }^{1}$, MIN DAI ${ }^{1}$, YU ZHANG ${ }^{1}$, QI WEI ${ }^{1}$ and FANYI MENG ${ }^{1,2}$ \\ ${ }^{1}$ Department of Hematology, Nanfang Hospital, Southern Medical University, Guangzhou, Guangdong 510515; \\ ${ }^{2}$ Department of Hematology, Kanghua Hospital, Dongguan, Guangdong 523000, P.R. China
}

Received February 5, 2015; Accepted June 27, 2015

DOI: $10.3892 / \mathrm{mco} .2015 .605$

\begin{abstract}
Decitabine (5-aza-2'-deoxycytidine; DAC) is a well-tolerated alternative to aggressive chemotherapy for leukemia, which induces differentiation and apoptosis of leukemic cells as a DNA hypomethylating agent. The aim of the present study was to investigate the feasibility of DAC sequentially combined with chemotherapy to reverse drug resistance. HL-60/ADR multidrug-resistant leukemia cells cultured in 96-well plates were pretreated with DAC for $72 \mathrm{~h}$; varying concentrations of aclacinomycin (ACLA) were then added to the wells, cell proliferation was tested using the Cell Counting Kit-8 assay, and DNA methyltransferase 1 (DNMT1) protein expression was detected by western blot analysis. Furthermore, we analyzed the therapeutic efficacy in 7 patients with high-risk acute myeloid leukemia (AML) receiving induction therapy with DAC sequentially combined with cytarabine, ACLA and granulocyte-colony stimulating factor (CAG regimen). The proliferation inhibition rate of HL-60/ADR cells treated with DAC at concentrations of 0.5 and $1.0 \mu \mathrm{mol} / 1$ sequentially combined with ACLA was significantly higher compared with that with ACLA alone $(\mathrm{P}<0.001$ for both). DNMT1 expression was significantly repressed following treatment with $1.0 \mu \mathrm{mol} / 1 \mathrm{DAC}$. Of the 11 patients, 8 (72.7\%) received induction therapy with DAC sequentially combined with CAG agents and achieved complete remission (CR) after 2 cycles of treatment; however, 3 (27.3\%) patients did not achieve remission. Myelosuppression was observed in all 11 patients and pulmonary infections developed in 9 patients (81.8\%) during the course of the study. At the last follow-up, 7 of the 8 patients who achieved CR
\end{abstract}

Correspondence to: Professor Fanyi Meng, Department of Hematology, Nanfang Hospital, Southern Medical University, 1838 Dadao North Street, Guangzhou, Guangdong 510515, P.R. China

E-mail: mengfu@medmail.com.cn

*Contributed equally

Key words: decitabine, CAG regimen, HL-60/ADR cells, high-risk acute myeloid leukemia remained in remission. The median follow-up was 6 months (range, 3-18 months). Therefore, pretreatment with DAC may increase the sensitivity of HL-60/ADR cells to ACLA via the epigenetic modulation of demethylation and the sequential administration of DAC and CAG regimen appears to be safe and effective for the treatment of patients with high-risk AML.

\section{Introduction}

Over the past several decades, improvements in chemotherapeutic agents and supportive care have resulted in significant progress in the treatment of patients with acute myeloid leukemia (AML). However, the treatment options for high-risk AML patients, particularly elderly, relapsed and refractory patients, remain limited. The outcomes of these patients are generally poor due to drug resistance, short durability of response, poor performance status and serious comorbidities following standard dose-intensive therapy $(1,2)$.

It was confirmed that epigenetic changes are crucial for the progression of numerous human neoplasms (3), as they are a universal mechanism of gene inactivation in malignant cells, possibly exceeding mutational events (4). Aberrant DNA methylation in gene promoters has been demonstrated to accompany these epigenetic changes and is also essential for the maintenance of altered gene expression status in malignant cells (5). Over the last few years, a growing number of studies have reported that leukemia is also characterized by high degrees of epigenetic changes $(6,7)$. These observations have led to the renewed interest in therapeutic regimens targeting the aberrant epigenome of cancer cells (8) and DNA methylation inhibitors are expected to be used as antineoplastic agents (3).

Decitabine (5-aza-2'-deoxycytidine; DAC), a DNA hypomethylating agent that induces differentiation and apoptosis of leukemic cells, is a well-tolerated alternative to aggressive chemotherapy. In a study published by Kantarjian et al (9), the efficacy and safety of different therapeutic regimens was compared in 485 elderly patients with newly diagnosed AML; the complete remission (CR) rate, including $\mathrm{CR}$ with delayed platelet recovery [CRp; platelet (PLT) count $\left.<100 \times 10^{9} / 1\right]$ was $17.8 \%$ with DAC vs. $7.8 \%$ with supportive care or cytarabine (Ara-C), without significant differences in safety. However, DAC monotherapy was associated with a relatively 
low rate of CR in AML $(10,11)$. Several groups have attempted to increase the response rate of DAC-based therapy by developing combination treatments (12-14). The aim of the present study was to investigate the effect of DAC sequentially combined with chemotherapeutic drugs in the HL-60/ADR multidrug-resistant leukemia cell line and retrospectively analyze the therapeutic efficacy in 7 high-risk AML patients.

\section{Materials and methods}

Reagents. The Cell Counting Kit-8 (CCK-8) was purchased from Dojindo Laboratories (Tokyo, Japan). DAC was supplied and formulated by Pharmachemie B.V. (Haarlem, The Netherlands). Aclacinomycin (ACLA) was purchased from Shenzhen Main Luck Pharmaceuticals Inc. (Shenzhen, China). Rabbit monoclonal anti-DNA methyltransferase 1 (DNMT1) antibody (dilution, 1:1,000; cat. no. 5032) and rabbit monoclonal anti-GAPDH antibody (dilution, 1:1,000; cat. no. 5174) and cell lysis buffer were purchased from Cell Signaling Technology, Inc. (Beverly, MA, USA). Polyvinylidene fluoride membranes were purchased from Millipore (Billerica, MA, USA).

Cell culture. The HL-60/ADR human AML cell line, a multidrug-resistant leukemia cell line, was obtained from the Institute of Hematology and Blood Diseases Hospital, Chinese Academy of Medical Sciences (Beijing, China). The cells were grown in RPMI-1640 (Invitrogen Life Technologies, Carlsbad, CA, USA) supplemented with $10 \%$ fetal bovine serum (Invitrogen Life Technologies) in plastic tissue culture plates in a humidified atmosphere containing $5 \% \mathrm{CO}_{2}$ at $37^{\circ} \mathrm{C}$.

Quantification of cell proliferation using the CCK-8 assay. For the growth inhibition assay, HL-60/ADR cells were cultured at a density of $10^{5}$ cells $/ \mathrm{ml}$ and aliquots $(100 \mu \mathrm{l})$ per well of the cell suspension were dispensed into 96-well plates. At $24 \mathrm{~h}$ after plating, DAC was added to the wells at concentrations of 0.5 and $1.0 \mu \mathrm{M}$. The plates were incubated in a humidified incubator in $5 \% \mathrm{CO}_{2}$ for $72 \mathrm{~h}$ at $37^{\circ} \mathrm{C}$. Subsequently, ACLA at varying concentrations was added to the wells. The cell proliferation was determined using the CCK- 8 at $24 \mathrm{~h}$ after dosing. The plates were then analyzed on an enzyme-linked immunosorbent assay plate reader (Bio-Rad 680; Bio-Rad, Hercules, CA, USA) at $490 \mathrm{~nm}$. All the experiments were performed in triplicate in at least 3 independent experiments.

Western blot analysis. Following treatment with $1 \mu \mathrm{mol} / 1$ DAC for $72 \mathrm{~h}$, the HL-60/ADR cells were harvested and lysed in cell lysis buffer. The proteins were separated by $10 \%$ sodium dodecyl sulphate-polyacrylamide gel electrophoresis and transferred onto polyvinylidene fluoride membranes. The membranes were blocked with $5 \%$ skimmed milk and incubated overnight at $4^{\circ} \mathrm{C}$ with anti-DNMT1 and anti-GAPDH antibodies in Tris-buffered saline $[10 \mathrm{~mm}$ Tris- $\mathrm{HCl}(\mathrm{pH} 8.0)$, $150 \mathrm{~mm} \mathrm{NaCl}$ ] with $0.1 \%$ Tween-20. Following incubation with peroxidase-conjugated secondary antibodies for $2 \mathrm{~h}$, the blots were developed using enhanced chemiluminescence (Molecular Imager ChemiDoc ${ }^{\mathrm{TM}}$ XRS; Bio-Rad).

Patients and treatment protocols. Following approval of the study protocol by the Institutional Review Board of
Nanfang Hospital we retrospectively analyzed 11 high-risk AML patients who were diagnosed according to the World Health Organization criteria (15) between May, 2012 and November, 2014 at the Department of Hematology of Nanfang Hospital. The pretreatment characteristics of the 11 patients are presented in Table I. All the patients received induction therapy with DAC sequentially combined with CAG agents, which consisted of DAC at a dose of $20 \mathrm{mg} / \mathrm{m}^{2}$ intravenously over $1 \mathrm{~h}$ daily on days 1-3, ACLA $10 \mathrm{mg}$ by intravenous infusion daily on days 4-10 or 13 and Ara-C $25 \mathrm{mg}$ subcutaneously twice daily on days $4-13$ or 17 . On days $4-13$ or 17 , granulocyte-colony stimulating factor (G-CSF) $300 \mu \mathrm{g}$ subcutaneously daily preceded the chemotherapeutic injections by $\sim 4-6 \mathrm{~h}$ and was discontinued when the white blood cell count reached $>20 \times 10^{9} / 1$. The treatment protocols for patients following achievement of CR were not uniform, due to differences in financial conditions (Table I). Complete blood count, hepatic and renal function tests and electrolyte levels were tested once or twice weekly during the drug administration period and marrow aspirates were monitored prior to and 2 weeks after treatment, to observe the therapeutic efficacy. G-CSF was used during the myelosuppression period and antibiotic therapy was administered as clinically indicated.

Response criteria and side effects. Response was assessed based on the criteria of the International Working Group for Diagnosis, Standardization of Response Criteria, Treatment Outcomes and Reporting Standards for Therapeutic Trials in Acute Myeloid Leukemia (16). CR required an absolute neutrophil (NEU) count of $\geq 1 \times 10^{9} / 1$, PLT count of $\geq 100 \times 10^{9} / 1$, marrow blasts $\leq 5 \%$ and disappearance of all signs and symptoms related to disease. CRp was defined as CR with a PLT count of $<100 \times 10^{9} / 1$. Any other response was considered as treatment failure. Neutropenia and thrombocytopenia was defined as NEU count $<0.5 \times 10^{9} / 1$ and PLT count $<20 \times 10^{9} / 1$, respectively. The reported non-hematological side effects included nausea, vomiting, diarrhea, skin rashes, liver or renal dysfunction and fever. The febrile episodes included fever of unknown origin and documented infections. The adverse drug reactions were scored according to the National Cancer Institute Common Toxicity Criteria, version 2.0 (17).

Statistical analysis. The statistical significance of the differences between the proliferation inhibition rate with DAC at two different concentrations sequentially combined with ACLA and the control group was assessed by one-way analysis of variance using the SPSS 13.0 software program (SPSS Inc., Chicago, IL, USA). P<0.05 was considered to indicate a statistically significant difference.

\section{Results}

Response of HL-60/ADR cells treated by DAC sequentially combined with ACLA. To evaluate the effects of sequentially combining DAC and ACLA on HL-60/ADR cell viability, the cells were treated with DAC $(0.5$ and $1.0 \mu \mathrm{M}$ for $72 \mathrm{~h})$ and sequentially with different concentrations of ACLA (0.1, $0.2,0.4,0.8,1.6$ and $3.2 \mu \mathrm{g} / \mathrm{ml}$ for $24 \mathrm{~h}$ ). The percentages of live/viable cells in the treated plates were measured using the CCK- 8 proliferation assay. The growth inhibition rate with 


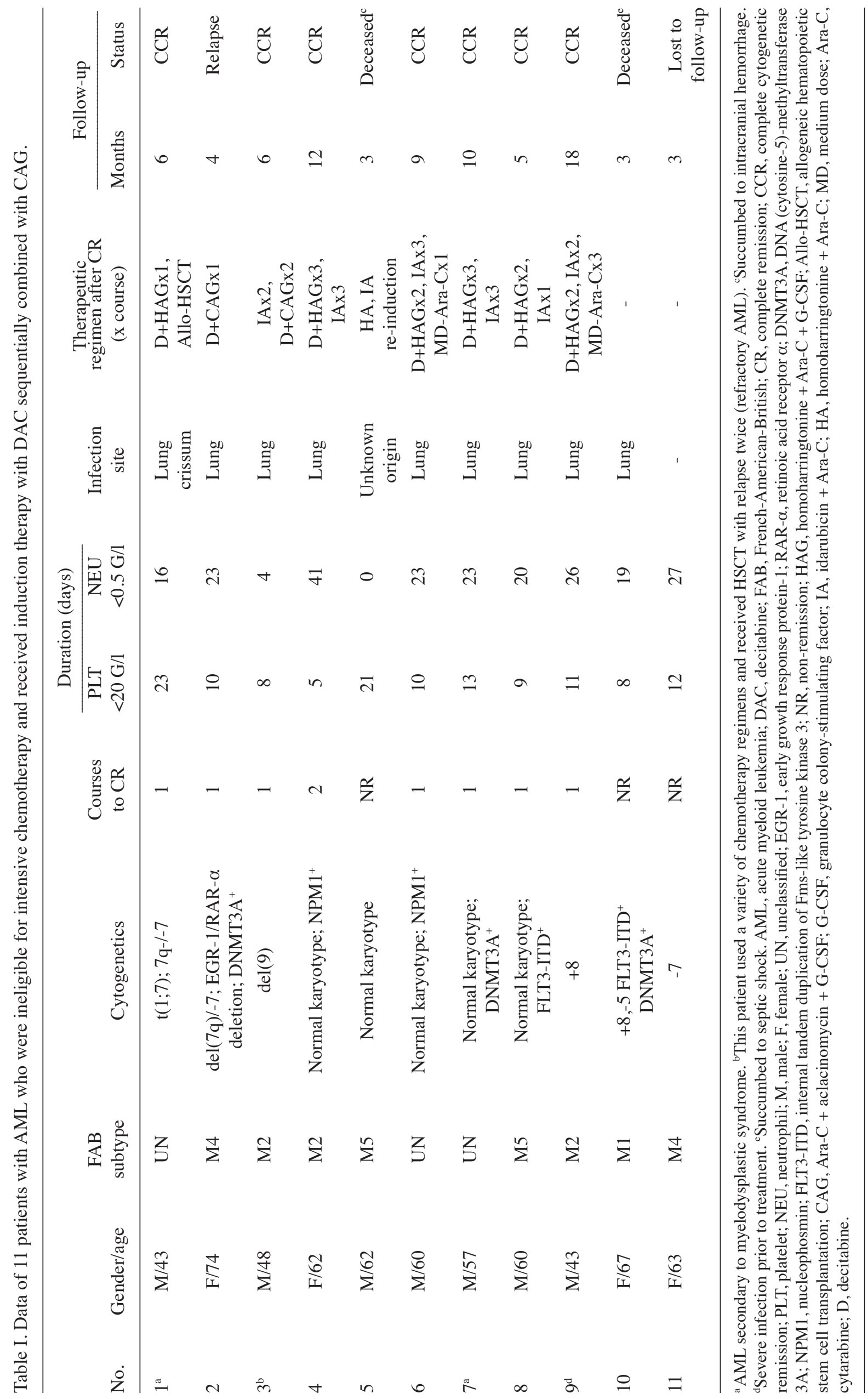


HL-60/ADR

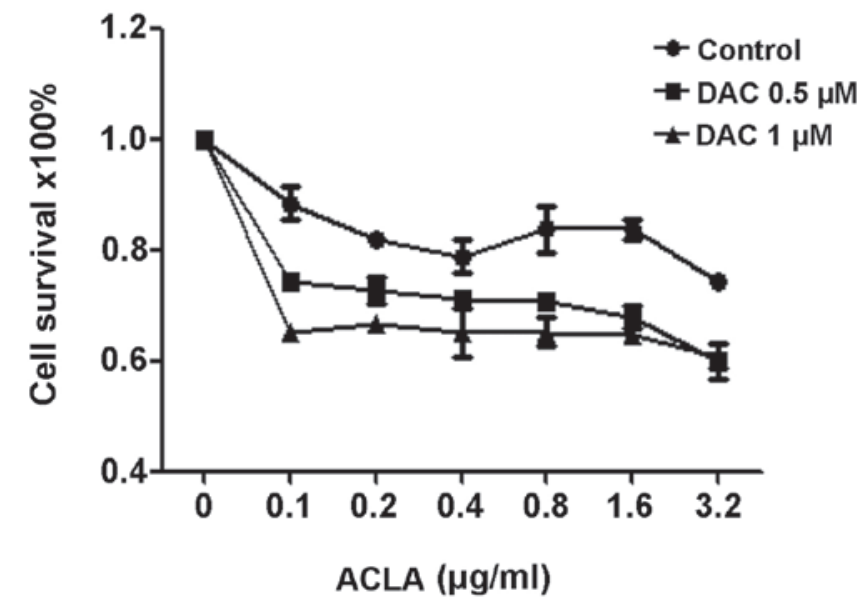

Figure 1. Sensitivity of HL-60/ADR cells to ACLA following pretreatment with DAC for $72 \mathrm{~h}$. ACLA, aclacinomycin; DAC, decitabine.

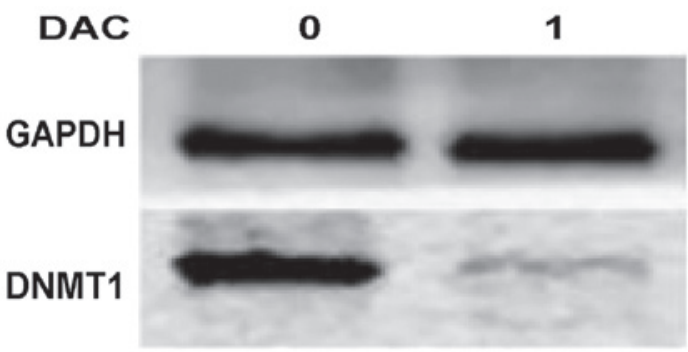

Figure 2. DNMT1 protein expression in HL-60/ADR cells after $72 \mathrm{~h}$ of treatment with $1.0 \mu \mathrm{mol} / 1 \mathrm{DAC}$, and in the control group. DNMT1, DNA methyltransferase 1; DAC, decitabine.

DAC at the two different concentrations sequentially combined with ACLA was significantly higher compared with that in the control group ( $\mathrm{P}<0.001$ for both, Fig. 1). The data revealed that the proliferation of HL-60/ADR cells was significantly inhibited when combining DAC and ACLA sequentially, compared with the treatment groups using ACLA alone.

DNMT1 protein expression in HL-60/ADR cells. DNMT1 protein expression was analyzed in HL-60/ADR cells following treatment with $1.0 \mu \mathrm{mol} / \mathrm{l}$ DAC for $72 \mathrm{~h}$. The western blot analysis demonstrated that DNMT1 expression was significantly repressed following treatment with $1.0 \mu \mathrm{mol} / \mathrm{l} \mathrm{DAC}$ in HL-60/ADR cells (Fig. 2).

Response to treatment. The therapeutic effects in the 11 patients are detailed in Table I. The median follow-up time was 6 months (range, 3-18 months) and the median course of consolidation chemotherapy after CR was 5 months (range, 1-7 months). Of the 11 patients, 7 (63.6\%) achieved CR after one treatment cycle and 1 patient $(9.1 \%)$ achieved CR after two cycles. Of the 8 patients achieving $\mathrm{CR}, 1$ patient underwent allogeneic hematopoietic stem cell transplantation after one cycle of consolidation therapy with DAC sequentially combined with HAG. One patient discontinued therapy after one cycle of consolidation therapy and did not receive any subsequent chemotherapy until he relapsed 4 months later, at which time re-induction with the original induction regimen was unsuccessful (non-remission; NR). A total of 6 patients (5 who achieved CR after one cycle and 1 who achieved CR after two cycles) received a total of 3-6 courses of consolidation therapy with DAC combined with CAG or homoharringtonine + Ara-C + G-CSF (HAG) and IA [idarubicin (IDA) + Ara-C] regimens. The patients remained in remission at the last follow-up. Two patients exhibited NR and succumbed to leukemia-related intracranial hemorrhage and septic shock. One patient was discharged from the hospital due to therapy discontinuation.

Side effects. Myelosuppression was observed in all the patients (Table I). The median duration of neutropenia was 23 days (range, $0-41$ days) and of thrombocytopenia 10 days (range, 5-23 days). During the myelosuppression period, a febrile episode of unknown origin was reported in 1 patient and pulmonary infections developed in 9 patients, but the symptoms were controlled following treatment with hematopoietic stimulating factor and symptomatic antibiotic support treatment. A total of 3 patients developed nausea and vomiting. Extramedullary toxicity was generally mild, without grade $\geq 2$ hepatic or renal dysfunction. Of the 8 patients who achieved $\mathrm{CR}, 6$ patients receiving consolidation therapy with sequential combination of DAC and CAG or HAG were analyzable for myelosuppression. The median time to NEU $<0.5 \times 10^{9} / 1$ was 8 days (range, $0-10.3$ days) and to PLT $<20 \times 10^{9} / 16$ days (range, 0-7 days). The duration of myelosuppression during consolidation therapy was shorter compared with that during the induction period.

\section{Discussion}

Although DAC has been found to be clinically effective as a single agent in patients with AML, the overall response rate (ORR) is relatively low, reportedly ranging between 8.5 and $26 \%(10,16)$. In order to improve the curative effect without increasing the toxicity, a therapeutic regimen using DAC combined with other antileukemic drugs has entered the stage of experiments in vitro and clinical studies. A number of hypomethylating combination trials for AML are underway and include the use of all trans-retinoic acid, Ara-C, IDA, daunorubicin (DNR), ACLA, thalidomide and homoharringtonine $(12,19-21)$. In our study, treatment with DAC prior to ACLA inhibited the proliferation of cultured HL-60/ADR multidrug-resistant cells.

Li et al (12) reported that the sequential combination of DAC and IDA induced synergistic cell death in U937 cells and AML cells isolated from AML patients, while tumor growth inhibition with this sequential combination was found to be higher compared with single-agent treatment or controls in vivo. However, other anthracyclines, including DNR and ACLA, did not exert a synergistic effect when sequentially combined with DAC, which was not consistent with our results. Sequential treatment with DAC and Ara-C was found by Leonard et al (19) to be more effective in reducing tumor burden compared with treatment with Ara-C alone in xenograft models of childhood AML.

Clinically, several data also investigated the strategy of hypomethylating agent-based combinations using DAC. A study by Zhang et al (22) compared the clinical efficacy and 
adverse reactions between low-dose DAC combined with CAG and CAG alone in intermediate- to high-risk myelodysplastic syndrome, and found that the CR rate was higher (75\%) in patients treated by the combination regimen compared with CAG alone (50\%), although the difference was not statistically significant. Jing et al (23) found that DAC combined with modified $C A G$ regimen for relapsed and refractory AML patients with AML1-ETO ${ }^{+}$displayed higher CR rate and fewer side effects. Benton et al (24) conducted a phase 1 study of patients with relapsed/refractory acute lymphocytic leukemia treated with DAC alone or in combination with the hyper-CVAD regimen; the ORR (including $\mathrm{CR}, \mathrm{CRp}$ and marrow $\mathrm{CR}$ ) was $21 \%$ in the DAC alone group and $56 \%$ in the combination regimen group, and certain patients, who had previously developed disease progression on Hyper-CVAD alone, achieved a CR when DAC was added. In the present study, we presented a retrospective analysis of a single-institution experience with the therapeutic efficacy of DAC sequentially combined with CAG in newly diagnosed patients with AML who were considered unfit for intensive chemotherapy. All the patients were monitored after treatment for the development of thrombocytopenia, neutropenia and infection, which were controllable. There was no reported drug-related mortality. The results were consistent with those reported by Bhatnagar et al (25).

These findings suggest that DAC sequentially combined with chemotherapy may enhance the antileukemic effect in vitro, as well as in clinical trials. Our results also confirmed that pretreatment with DAC may act as a potential sensitizer to ACLA, thereby improving its curative efficacy via the epigenetic modulation of demethylation in HL-60/ADR cells and AML patients.

Multiple mechanisms may be involved in this phenomenon. Aberrant expression of DNMTs, which promote DNA methylation, is recognized as a key factor in the onset of cancer and drug resistance $(26,27)$. DNMT1 is crucial for the maintenance of the methylation landscape due to its ability to recognize hemimethylated DNA and conserve methylation during somatic cellular division (27). High levels of DNMT1 expression have been reported in cancer patients who are not responsive to chemotherapy (26). DAC incorporated into DNA covalently binds to DNMT1, leading to the reduction of available DNMT1 protein in cells, which in turn results in DNA demethylation and expression of methylation-silenced genes $(28,29)$. In the present study, we demonstrated that DAC decreased DNMT1 protein expression levels, which was consistent with previous findings.

In conclusion, we retrospectively reported a single-institution experience using DAC sequentially combined with CAG in newly diagnosed high-risk AML patients. These findings suggest clinical potential in the sequential administration of DAC and CAG regimen for the treatment of elderly and relapsed/refractory AML patients, or patients with secondary AML. However, due to the limited number of cases, large-scale multicenter studies are required to confirm our results.

\section{References}

1. Kantarjian H, Ravandi F, O'Brien S, Cortes J, Faderl S, Garcia-Manero G, Jabbour E, Wierda W, Kadia T, Pierce S, et al: Intensive chemotherapy does not benefit most older patients (age 70 years or older) with acute myeloid leukemia. Blood 116 4422-4429, 2010.
2. Schiller GJ: When a gold standard is made of tin. Blood 116: 4386-4387, 2010.

3. Santini V, Kantarjian HM and Issa JP: Changes in DNA methylation in neoplasia: Pathophysiology and therapeutic implications. Ann Intern Med 134: 573-586, 2001

4. Baylin SB and Ohm JE: Epigenetic gene silencing in cancera mechanism for early oncogenic pathway addiction? Nat Rev Cancer 6: 107-116, 2006.

5. Jones PA and Takai D: The role of DNA methylation in mammalian epigenetics. Science 293: 1068-1070, 2001.

6. Issa JP, Baylin SB and Herman JG: DNA methylation changes in hematologic malignancies: Biologic and clinical implications. Leukemia 11 (Suppl 1): S7-S11, 1997.

7. Melki JR and Clark SJ: DNA methylation changes in leukaemia. Semin Cancer Biol 12: 347-357, 2002.

8. Egger G, Liang G, Aparicio A and Jones PA: Epigenetics in human disease and prospects for epigenetic therapy. Nature 429: 457-463, 2004.

9. Kantarjian HM, Thomas XG, Dmoszynska A, Wierzbowska A, Mazur G, Mayer J, Gau JP, Chou WC, Buckstein R, Cermak J, et al: Multicenter, randomized, open-label, phase III trial of decitabine versus patient choice, with physician advice, of either supportive care or low-dose cytarabine for the treatment of older patients with newly diagnosed acute myeloid leukemia. J Clin Oncol 30: 2670-2677, 2012

10. Issa JP, Garcia-Manero G, Giles FJ, Mannari R, Thomas D, Faderl S, Bayar E, Lyons J, Rosenfeld CS, Cortes J, et al: Phase 1 study of low-dose prolonged exposure schedules of the hypomethylating agent 5-aza-2'-deoxycytidine (decitabine) in hematopoietic malignancies. Blood 103: 1635-1640, 2004

11. Yang AS, Doshi KD, Choi SW, Mason JB, Mannari RK, Gharybian V, Luna R, Rashid A, Shen L, Estecio MR, et al: DNA methylation changes after 5-aza-2'-deoxycytidine therapy in patients with leukemia. Cancer Res 66: 5495-5503, 2006.

12. Li K, Hu C, Mei C, Ren Z, Vera JC, Zhuang Z, Jin J and Tong H: Sequential combination of decitabine and idarubicin synergistically enhances anti-leukemia effect followed by demethylating Wnt pathway inhibitor promoters and downregulating Wnt pathway nuclear target. J Transl Med 12: 167, 2014.

13. Gore SD, Baylin S, Sugar E, Carraway H, Miller CB, Carducci M, Grever M, Galm O, Dauses T, Karp JE, et al: Combined DNA methyltransferase and histone deacetylase inhibition in the treatment of myeloid neoplasms. Cancer Res 66: 6361-6369, 2006.

14. Blum W, Klisovic RB, Hackanson B, Liu Z, Liu S, Devine H, Vukosavljevic T, Huynh L, Lozanski G, Kefauver C, et al: Phase I study of decitabine alone or in combination with valproic acid in acute myeloid leukemia. J Clin Oncol 25: 3884-3891, 2007.

15. Vardiman JW: The World Health Organization (WHO) classification of tumors of the hematopoietic and lymphoid tissues: an overview with emphasis on the myeloid neoplasms. Chem-Biol Interact 184: 16-20, 2010.

16. Cheson BD, Bennett JM, Kopecky KJ, Büchner T, Willman CL, Estey EH, Schiffer CA, Doehner H, Tallman MS, Lister TA, et al; International Working Group for Diagnosis, Standardization of Response Criteria, Treatment Outcomes, and Reporting Standards for Therapeutic Trials in Acute Myeloid Leukemia: Revised recommendations of the International Working Group for Diagnosis, Standardization of Response Criteria, Treatment Outcomes and Reporting Standards for Therapeutic Trials in Acute Myeloid Leukemia. J Clin Oncol 21: 4642-4649, 2003.

17. Postma TJ and Heimans JJ: Grading chemotherapy-induced peripheral neuropathy. Ann Oncol 11: 509-513, 2000.

18. Lübbert M,Suciu S, Baila L, Rüter BH,Platzbecker U, Giagounidis A, Selleslag D, Labar B, Germing U, Salih HR, et al: Low-dose decitabine versus best supportive care in elderly patients with intermediate- or high-risk myelodysplastic syndrome (MDS) ineligible for intensive chemotherapy: Final results of the randomized phase III study of the European Organisation for Research and Treatment of Cancer Leukemia Group and the German MDS Study Group. J Clin Oncol 29: 1987-1996, 2011.

19. Leonard SM, Perry T, Woodman CB and Kearns P: Sequential treatment with cytarabine and decitabine has an increased anti-leukemia effect compared to cytarabine alone in xenograft models of childhood acute myeloid leukemia. PLoS One 9: e87475, 2014.

20. Peng CY, Jiang J, Zheng HT and Liu XS: Growth-inhibiting effects of arsenic trioxide plus epigenetic therapeutic agents on leukemia cell lines. Leuk Lymphoma 51: 297-303, 2010.

21. Qin T, Youssef EM, Jelinek J, Chen R, Yang AS, Garcia-Manero G and Issa JP: Effect of cytarabine and decitabine in combination in human leukemic cell lines. Clin Cancer Res 13: 4225-4232, 2007. 
22. Zhang YP, Wu WZ and Cui GX: Comparison of clinical efficacy between decitabine combined with CAG regimen and CAG regimen alone in patients with intermediate to high-risk myelodysplastic syndromes. J Exp Hematol 22: 1341-1344, 2014 (In Chinese).

23. Jing Y, Zhu CY, Zhang Q, et al:: Clinical efficacy of decitabine combined with modified CAG regimen for relapsed-refractory acute myeloid leukemia with AML1-ETO ${ }^{+}$. J Exp Hematol 22: 1245-1250, 2014 (In Chinese).

24. Benton CB, Thomas DA, Yang H, Ravandi F, Rytting M, O'Brien S, Franklin AR, Borthakur G, Dara S, Kwari M, et al: Safety and clinical activity of 5-aza-2'-deoxycytidine (decitabine) with or without Hyper-CVAD in relapsed/refractory acute lymphocytic leukaemia. Br J Haematol 167: 356-365, 2014.

25. Bhatnagar B, Duong VH, Gourdin TS, Tidwell ML, Chen C, Ning Y,Emadi A, Sausville EA and Baer MR: Ten-day decitabine as initial therapy for newly diagnosed patients with acute myeloid leukemia unfit for intensive chemotherapy. Leuk Lymphoma 55: 1533-1537, 2014
26. Mutze K, Langer R, Schumacher F, Becker K, Ott K, Novotny A, Hapfelmeier A, Höfler H and Keller G: DNA methyltransferase 1 as a predictive biomarker and potential therapeutic target for chemotherapy in gastric cancer. Eur J Cancer 47: 1817-1825, 2011.

27. Robert MF, Morin S, Beaulieu N, Gauthier F, Chute IC, Barsalou A and MacLeod AR: DNMT1 is required to maintain CpG methylation and aberrant gene silencing in human cancer cells. Nat Genet 33: 61-65, 2003.

28. Daskalakis M, Blagitko-Dorfs N and Hackanson B: Decitabine. Recent Results Cancer Res 184: 131-157, 2010.

29. Oki Y, Aoki E and Issa JP: Decitabine - bedside to bench. Crit Rev Oncol Hematol 61: 140-152, 2007. 